\title{
AFECTIVIDAD Y COMPETENCIA EXISTENCIAL EN ESTUDIANTES DE ESPAÑOL COMO LENGUA EXTRANJERA EN BRASIL*
}

\author{
AFFECTIVE AND EXISTENTIAL COMPETENCES IN STUDENTS \\ OF SPANISH AS A FOREIGN LANGUAGE IN BRAZIL
}

\author{
PAULO ROBERTO MARINS DE ANDRADE \\ Universidad de Granada, España \\ Agencia Española para la Cooperación Internacional y el Desarrollo (AECID) \\ pablo.and@uol.com.br \\ JUAN RAMÓN GUIJARRO OJEDA \\ Universidad de Granada, España \\ jrgo@ugr.es
}

\section{RESUMEN}

El presente artículo tiene como objetivo el estudio de los componentes afectivos de la competencia existencial en estudiantes de español como lengua extranjera en un contexto de no-inmersión en un Centro Superior de Lenguas en el Distrito Federal de Brasilia. Serán planteadas variables como: motivación, autoestima, autonomía, ansiedad y empatía y su relación con el aprendizaje de lenguas extranjeras. Cuestiones tan relevantes en lingüística aplicada como la relación entre emociones y cognición, así como la inteligencia emocional serán abordadas en el estudio empírico al preguntarle al alumnado cuestiones relativas sobre cómo son trabajados estos contenidos en la clase de E/LE. El análisis de los resultados obtenidos nos proporcionará elementos formativos valiosos que nos ayudarán a replantear las prácticas docentes en el área de estudio.

Palabras claves: Español como lengua extranjera, competencia existencial, afectividad.

\section{ABSTRACT}

In this paper we address the study of the affective component of the existential competence in students of Spanish as a Foreign Language in a non-immersion context in a Language

\footnotetext{
* Este estudio se contextualiza en el marco general de investigación del Proyecto I+D+i "Implementación de un modelo basado en la conciencia de la identidad docente y la competencia existencial en la formación del profesorado de lengua extranjera" SEJ 2007-62329, financiado por el Ministerio de Ciencia e Innovación del Gobierno de España y desarrollado en la Universidad de Granada (España).
} 
School in Brasilia. Aspects such as motivation, self-esteem, autonomy, anxiety, and empathy and their relationship to the learning of foreign languages are stated in this research report. Core concepts in Applied Linguistics such as the relationship between emotion and cognition or emotional intelligence are considered in the empirical study when we ask students how these topics are stimulated in the S/FL classroom. The analysis of results will provide us with valuable formative elements to help us to rethink our teaching practices in the foreign language area.

Keywords: Spanish as a Foreign Language, existential competence, affective variables.

Recibido: 16-10-2009. Aceptado: 22-03-2010.

\section{INTRODUCCIÓN}

- 1 desarrollo de este trabajo viene motivado por la importancia que en el ámCbito de las lenguas extranjeras tiene la relación entre afectividad y cognición. La eficacia de las destrezas cognitivas está condicionada por el estado emocional del aprendiz, lo que requiere un mayor autocontrol y autoconocimiento por parte del alumnado para poder resultar en un aumento de su autoestima y su automotivación.

Este artículo se estructura siguiendo dos ejes bien definidos e interrelacionados. Por un lado, se presenta una exposición teórica sobre la importancia de las emociones y la cognición en el aprendizaje de una lengua extranjera, de lo que se deriva la competencia existencial tal y como ha sido conceptualizada por el Consejo de Europa (2002). Dentro de esta competencia se hacen relevantes aspectos como la motivación, la ansiedad, la autoestima, la empatía o la autonomía que discutimos en este trabajo. Por otro lado, presentamos la implementación, análisis y conclusiones de un estudio empírico realizado con alumnado de Español como Lengua Extranjera de un Centro Superior de Lenguas en Brasilia. Dicho estudio está basado en los planteamientos teóricos anteriomente mencionados para tratar de arrojar luz sobre las percepciones de este alumnado en torno a la relevancia y significatividad de los factores afectivo-existenciales en su propio desarrollo académico de la lengua extranjera en cuestión.

Los resultados de este estudio nos sirven para arrojar luz sobre el cómo y porqué de determinadas actitudes de estudiantes en un contexto de aprendizaje de español como lengua extranjera (E/LE) de no-inmersión y comprender de manera más profunda cómo el estudiantado maneja o desea manejar los factores internos para obtener un desempeño mejor en su aprendizaje. 


\section{QUÉ ENTENDEMOS POR COMPETENCIA EXISTENCIAL}

Para utilizar la lengua en sus variados contextos de manera eficaz hace falta que el usuario, alumno o alumna, esté capacitado para hacerlo por medio de la utilización de competencias y, por tanto, será necesario desarrollar sus características individuales, a saber, sus rasgos de carácter, sumamente importantes en el proceso de enseñanza y aprendizaje de la lengua extranjera. En definitiva, para que el acto de comunicación se realice con eficacia es necesario que los aprendices utilicen un amplio espectro de competencias tanto lingüístico-comunicativas como de índole personal.

El Consejo de Europa (2002) define y diferencia en el Marco Común Europeo de Referencia para las Lenguas (MCERL) una serie de competencias requeridas que deben ser desarrolladas por el alumno para poder enfrentarse a diferentes situaciones comunicativas. Las competencias del/de la estudiante se dividen en dos grandes grupos: "competencias generales" y "competencias lingüísticas" que se interrelacionan formando un todo. La competencia existencial es una de las subcompetencias de las "competencias generales", las cuales, de manera transversal, contemplan otros ámbitos como el de la cultura, la conciencia intercultural y las habilidades y destrezas metacognitivas.

La competencia existencial aglutina el componente actitudinal, el componente motivacional, el componente axiológico y los estilos cognitivos/estilos de aprendizaje (Guijarro 2007). En otras palabras, el "saber ser" se desarrolla a través del autoconocimiento y del saber socializarse en diferentes contextos culturales y, por ende, comunicativos. Es la capacidad de desarrollar actitudes positivas hacia la lengua y cultura hispánicas, desarrollar actitudes que favorezcan el aprendizaje, además de la capacidad para desarrollar la motivación (Centro Virtual Cervantes, 2008). El MCERL (2002) establece que las actitudes y los factores de personalidad de los alumnos también inciden en su "capacidad para aprender" (competencia general), la cual se relaciona directamente con los conceptos de "autonomía" y de "estrategias de aprendizaje". De ahí que el fomento de la competencia existencial gane terreno como objetivo principal en la enseñanza de lenguas. Por todo ello, se infiere que esta competencia guarda una estrecha relación con lo que se conoce tradicionalmente como el componente afectivo en la enseñanza de lenguas.

El siguiente esquema-resumen nos ofrece un espectro detallado de todos los elementos o factores que englobaría la competencia existencial (saber ser/savoir être) (Consejo de Europa, 2002):

- Actitudes: apertura hacia nuevas experiencias, otras personas, ideas, pueblos, sociedades y culturas, y el interés que muestran hacia ello; voluntad de relativizar la propia perspectiva cultural y el propio sistema de valores culturales; voluntad y capacidad de distanciarse de las actitudes convencionales en cuanto a la diferencia cultural. 
- Motivaciones: intrínsecas y extrínsecas; instrumentales e integradoras; impulso comunicativo, la necesidad humana de comunicarse.

- Valores: por ejemplo, éticos y morales.

- Creencias: por ejemplo, religiosas, ideológicas, filosóficas.

- Estilos cognitivos: convergente y divergente; holístico, analítico y sintético.

- Factores de personalidad: por ejemplo, locuacidad y parquedad; espíritu emprendedor e indecisión; optimismo y pesimismo; introversión y extraversión; actividad y pasividad; personalidad con complejo de culpabilidad, acusadora y disculpadora; el miedo y la vergüenza, o la personalidad liberada de ellos; rigidez y flexibilidad; mentalidad abierta y mentalidad cerrada; espontaneidad y autocontrol; grado de inteligencia; meticulosidad y descuido; capacidad memorística; diligencia y pereza; ambición y conformismo; autoconciencia y falta de autoconciencia; independencia y falta de independencia; seguridad en sí mismo y falta de seguridad; autoestima y falta de autoestima.

\section{FACTORES AFECTIVO-EMOCIONALES QUE CONDICIONAN LA ENSEÑANZA Y APRENDIZAJE DE E/LE}

Las condiciones afectivo-emocionales del profesorado y alumnado inciden directamente en el aprovechamiento académico y, por consiguiente, en el proceso de enseñanza educativa eficaz. De este modo, consideramos que los factores emocionales se dividen, básicamente, en dos grupos: emociones negativas y positivas. No obstante, según Goleman (1997), la mayoría de las emociones serían consideradas, por lo general, negativas y, consecuentemente, perniciosas para la enseñanza/ aprendizaje de una lengua extranjera. Además, de acuerdo a Goleman, (1997: 34) "las emociones dolorosas suelen hacer que enfermemos y los estados mentales saludables tienden a fomentar la salud". Por todo ello, coincidimos con Arnold y Brown (2000) al afirmar que el hecho de estimular la empatía, la autoestima, la motivación y otras variables emocionales positivas facilita notablemente el proceso de aprendizaje de un idioma extranjero.

\subsection{La motivación}

La motivación es uno de los factores principales que influyen en el aprendizaje. Cuando se trata del aprendizaje de lenguas extranjeras, esta variable aún se torna más importante, sobre todo si no se trabaja en un contexto de inmersión. A pesar de estar relacionada esta variable tanto con estudiantes como con docentes, se suele dar mayor énfasis a la motivación del estudiante. La motivación es un ámbito psico-educativo muy extenso y controvertido, ya que tiene que ver con la personalidad de cada individuo, pero también depende de la metodología adoptada por 
el/la docente y si ésta está establecida conforme a las necesidades y expectativas de su alumnado (Guijarro, 2004).

Según Williams y Burden (1999: 128), la palabra motivación puede ser representada por "un estado de activación cognitiva y emocional", además de estar determinada también por otros factores como el interés, la curiosidad o el logro, todos distintos según diferentes situaciones.

La motivación puede depender de factores externos (influencia de otras personas o de algún suceso -motivación extrínseca-) o internos (interés o curiosidad -motivación intrínseca-). Por otro lado, para Gardner (2007: 7), a pesar de estar claras y ser compensatorias las ventajas que conlleva el aprender un idioma extranjero, no son estrictamente necesarias en general en la vida cotidiana pues existen otras prioridades a nivel de supervivencia. Así es que el carácter altamente competitivo del sistema educativo o el miedo al fracaso podrían constituir un importante obstáculo para lograr el éxito.

Por otro lado, es muy complejo intentar descubrir qué es lo que crea la motivación. Es sabido que es función del profesor o profesora de idiomas despertar el interés de los/las aprendices, establecer objetivos y proveer los medios para lograr estos objetivos. Para Chomsky (1998 apud Arnold y Brown 2000: 30) el $99 \%$ aproximadamente de la enseñanza se destina a lograr que los alumnos se interesen por el material. Así es que es primordial el papel del profesor como agente motivador de sus estudiantes - primicia de la "competencia existencial"- así como "intermediador" entre los discentes y su material de estudio. De la misma manera, el propio profesor debe estar motivado para poder transmitirles este sentimiento: "Si un profesor no está motivado, si no ejerce de forma satisfactoria su profesión, es muy difícil que sea capaz de motivarlos” (Alonso Tapia y Cartula Fita, 2003: 85).

\subsection{La ansiedad}

La ansiedad es una emoción dolorosa que puede condicionar de forma negativa el aprendizaje de idiomas si no se encuentra en un nivel controlable. Según Gardner y MacIntyre (1993), la ansiedad en la clase de idiomas es el temor o la aprensión que surgen cuando un alumno tiene que realizar una actividad en una segunda lengua extranjera. No obstante, como estado pasajero, tiende a disminuir con el tiempo, pero, si de pronto se convierte en un rasgo permanente, puede tener efectos generalizados a lo largo del proceso de aprendizaje. Hablando del lenguaje, para Oxford (2000: 80), la ansiedad se relaciona con aspectos que van desde los muy personales (como la autoestima) hasta los relacionados con procedimientos, actividades y prácticas del aula.

Algunos investigadores afirman que existe una modalidad positiva de ansiedad - "ansiedad facilitadora"- en el aprendizaje de idiomas pues mantiene alerta al alumnado. Por el contrario, gran parte de las investigaciones lingüísticas demues- 
tran una relación negativa entre ansiedad y ejecución -"ansiedad debilitadora"-. Por ejemplo, para Horwitz (1986) la ansiedad sólo es útil para tareas muy elementales de aprendizaje, pero no lo es cuando se trata de un aprendizaje más complicado, como el de idiomas. Sin embargo, Omaggio Hadley (2000) sugirió que un cierto grado de "tensión" podría ser útil para el aprendizaje de idiomas; declinó en denominar "ansiedad” a esta tensión. Ya Krashen (1982) mantenía que no hay ningún aspecto útil en la ansiedad respecto a la adquisición de lenguas, que siempre requiere por definición que la ansiedad sea cero. Por otro lado, la ansiedad útil podría servir para las tareas en situaciones académicas bajo una metodología más tradicional de aprendizaje de idiomas. Rebeca Oxford (2000: 30) afirma que la ansiedad útil se relaciona con:

- Un buen dominio del idioma y una elevada seguridad en sí mismo entre un grupo reducido de excelentes aprendices de idiomas;

- La producción oral de estructuras difíciles de inglés (por nativos de árabe y de español);

- Las buenas notas en clase de idiomas para estudiantes de francés, alemán y español en clases académicas (bajo métodos más tradicionales), pero no para alumnos/as de clases del método audiolingual.

\subsection{La autoestima}

Según Arnold y Brown (2000: 80), "la autoestima es la valoración que hacemos de nuestra propia valía o valor basada en sentimientos de eficacia, es el sentimiento de que interactuamos eficazmente con nuestro entorno". Así, es función del profesor el establecimiento de un ambiente emocional e intelectualmente seguro para propiciar el fomento de la autoestima. Waltz y Bleuer (1992) y Arnold y Brown (2000: 30) concluyen que "[...] los aspectos cognitivos del aprendizaje se fomentan en un ambiente que promueva la autoestima”. Los profesores no sólo deben ayudar a sus alumnos a mejorar su autoestima, sino también a trabajar la propia, pues esto también es transmitido a los alumnos (Arnold, 2000: 125). Por ende, para que la actividad cognitiva y afectiva tenga éxito es fundamental una buena dosis de autoestima tanto del estudiantado como del profesorado.

La autoestima es vulnerable cuando el/la estudiante se percibe a sí mismo como muy competente en su lengua materna y totalmente incapacitado o limitado respecto a la lengua meta (Price, 1991 apud Arnold y Brown, 2000). Horwitz y Cop (1986, apud Arnold y Brown, 2000) corroboran esta idea al afirmar que el aprendizaje de idiomas puede ser una "amenaza" para la autoestima al privar a los alumnos de sus "medios normales" (recursos lingüísticos / estrategias personales) de comunicación, de su libertad de cometer errores y de su capacidad para comportarse como personas normales. Así, los docentes tendrían que prestar atención a la 
tendencia de "artificializar" o "relativizar" demasiado los contextos de enseñanza/ aprendizaje de una lengua ignorando así, aún sin saberlo, las potencialidades de los alumnos y perjudicando la autoestima de éstos con el fin de "evitar el error". Este microcosmos del aula aún se torna más artificial si los alumnos siempre están asumiendo roles y expresando opiniones falsas o hipócritas en beneficio de la "armonía del ambiente de clase", sin tener en cuenta las emociones, valores, cualidades, sueños y aspiraciones de los mismos, los cuales deben ser respetados y valorados e, incluso, elevados a la categoría de "contenidos afectivos". Concluimos que la baja autoestima así como su desconsideración (la falta de un tratamiento adecuado) puede incluso bloquear el aprendizaje del alumno así como limitar su creatividad.

Uno de los procedimientos indicados para trabajar la autoestima con niños es el autoconocimiento (inteligencia intrapersonal), el conocimiento de otras personas (inteligencia interpersonal) y la comunicación eficaz: cómo escuchar mientras los demás hablan y las destrezas lingüísticas (Andrés, 2000: 108). Por esto, la autoestima estaría estrechamente vinculada con las relaciones sociales y con la aceptación de uno por los demás y por él mismo, lo que en repetidas veces no es fácil de lograr por falta de seguridad en las potencialidades y cualidades de uno o por no expresarlas por timidez. Para lograr trabajar conceptos como: singularidad, crecimiento, cambio, sentimientos, talento y habilidades, cooperación, amistad o comunicación se proponen actividades como: lluvia de ideas (brainstorming), círculo de sentimientos, hojas de actividades, trabajos manuales, lectura o escritura de canciones según nos sugiere Andrés (2000).

Peasoner (1992: 24, apud Andrés, 2000: 116) también afirma que el desarrollo del sentimiento "de felicidad" y de "bienestar" tendría efectos poco duraderos y que lo ideal sería fortalecer las fuentes internas de su autoestima, lo que, según su visión, sería más eficaz. Por otro lado, el profesor tendría que tener cuidado con centrarse demasiado en el incremento de la "autoestima" pues tal hecho podría también, inversamente, acarrear sentimientos de ensimismamiento o narcisismo, dificultando así los procesos internos del aula como la cooperación.

\subsection{La empatía}

Arnold y Brown (2000: 36) definen empatía como "el proceso de ponerse en la piel de otra persona". Es una identificación mental y afectiva de uno con respecto al estado de ánimo de otro. Para Arnold y Brown (2000: 36) "la empatía es un factor quizá el más importante que contribuye para la coexistencia harmoniosa de los individuos en la sociedad". Con este sentimiento podemos volver nuestra atención más detenidamente hacia el desarrollo de lo que se llama competencia intercultural, puesto que la empatía nos ayuda a fomentar nuestra conciencia colectiva hacia la diversidad además del respeto frente a otras formas de enseñar/ aprender (Trujillo Sáez, 2005). 
Es obvio que sólo puede desarrollar empatía el profesor que mira y trata a sus alumnos primeramente como seres humanos, frutos de determinado contexto socioeconómico y cultural y no meramente como números del "cuerpo discente". Arnold (2000) y Guijarro (2007) consideran que el aula de idiomas es un espacio no sólo para el estudio de la lengua extranjera, sino también una fuente para la educación en valores y para el desarrollo de la Inteligencia Emocional. Entonces, el primer trabajo del profesor humanista es el de enseñar a sus estudiantes a tomar conciencia de sus propios sentimientos y conocerlos para saber lidiar con ellos desarrollando así su Coeficiente Emocional y sólo así estaría más capacitado para "ponerse en el lugar del otro" (Hogan, 1969 apud Arnold y Brown, 2000: 36).

Ahora bien, queda descubrir qué tipos de actividades de aula se podrían utilizar para fomentar la empatía de los alumnos y qué enfoque/método sería el más proclive al desarrollo de la misma (Arnold y Brown, 2000). Creemos que para contestar a esta pregunta primero el docente debería hacer un sondeo en la clase para descubrir las preferencias, gustos y tipos de personalidad del alumnado y así poder elegir la actividad con la que más se identifican. Este es un trabajo que puede ir en contra de muchas de las creencias y valores del propio profesor, no obstante, es esencial para que éste pueda aprehender el lado emocional de sus estudiantes. Consideramos que aunque no se consiga lograrlo hay que buscar un punto de contacto con ellos. Y con relación a la elección del enfoque más proclive hacia la empatía, el profesor debería conocer cuáles son los motivos reales que llevaron a los estudiantes a interesarse por este idioma para poder elegir la metodología o actividades más apropiadas y así sentirán que su esfuerzo tiene un fin útil: el alcance de sus metas personales.

Para el perfecto desarrollo del ejercicio humanístico el/la docente, además de tener una fuerte conciencia del proceso grupal, del estado de ánimo y de cómo esto afecta al aprendizaje, debe trabajar el autoconocimiento y el origen de sus estudiantes. Y para presentar este comportamiento deberá hacer uso de la empatía, así como de la observación y escucha atenta si quiere que el ejercicio humanístico sea pertinente y que ofrezca a los alumnos una nueva experiencia.

Arnold (2000: 290) sugiere la utilización de la imaginación (“imágenes mentales") para desarrollar la empatía pues "utilizando nuestra imaginación y nuestra intuición podemos intentar percibir los sentimientos y los pensamientos de la otra persona” (Miller, 1981: 118 apud Arnold, 2000). Por lo tanto, la empatía es importante en el aprendizaje de idiomas porque supone el descubrimiento de otra cultura, desarrollando así la competencia intercultural.

\subsection{La autonomía}

Para Giovannini (2000: 24), según recientes estudios sobre las causas por las que un alumno es más eficaz que los demás en el aprendizaje, los "buenos" alumnos son aquellos que son eficaces, activos y que, simplemente, saben cómo aprender, 
es decir, que poseen sus propias estrategias de aprendizaje (estrategias del aprendiz) y un buen nivel de autonomía. Por otra parte, hay quien cree que no hay recetas para ser un buen estudiante y que cada uno debe desarrollar sus propias estrategias conociéndose a sí mismo, explotando sus habilidades y compensando sus deficiencias -competencia estratégica-. Para Giovannini (2000: 26) enseñar a los alumnos a desarrollar la propia autonomía ayudaría al estudiante a tomar conciencia de sus propios recursos y estrategias, ya que, por lo general, están "dormidos" o incluso "bloqueados por las experiencias, a veces negativas, del período escolar".

Es evidente que hay que respetar los estilos de aprendizaje y las diferencias individuales y de personalidad. Si el alumno o alumna no tiene ninguna vocación para aprender autónomamente, constituye cierta "violencia" forzarlo, pues estaríamos forzándolo a cambiar su propia personalidad en contra suya.

Para Giovannini (2000: 24), el alumno eficaz debe estar en condiciones de tomar una serie de decisiones necesarias para planificar y llevar a la práctica este programa, tales como el hecho de seleccionar los contenidos didácticos y lingüísticos para llegar a los objetivos previstos y ser capaz de autoevaluar las estrategias utilizadas y los resultados obtenidos. Se concluye que el eje central y la fuerza motora de la práctica de la autonomía se encuentra en la autoevaluación, pues es a partir de ella que el/la aprendiz tendría que tomar decisiones sobre lo que deberá cambiar en su forma de aprender (feedback). Hecha al inicio del curso es una forma para que uno evalúe sus propias necesidades, intereses y expectativas como costumbres de aprendizaje adquiridos en estudios anteriores.

Por todo ello, el incremento de la competencia comunicativa tiene una estrecha relación con el nivel de autonomía y con el "saber aprender" pues "cuanto mayor sea la competencia de aprendizaje, mayor será la competencia comunicativa del alumno para expresarse en español" (Giovannini, 2000: 28).

\section{METODOLOGÍA}

\subsection{Objetivos de la investigación}

Esta investigación tiene como objetivo estudiar, analizar y evaluar los resultados de algunos aspectos del proceso de enseñanza-aprendizaje de E/LE con relación a factores afectivo-emocionales desde el punto de vista del alumnado. Así se establecieron los siguientes objetivos específicos:

a. Conocer las opiniones del alumnado sobre aspectos afectivo-existenciales que intervienen en su aprendizaje de E/LE.

b. Conocer el grado en que variables afectivo-emocionales tales como el control de la ansiedad, el desarrollo de la autonomía y de la autoestima o la motivación pueden facilitar o entorpecer la solución de los desafíos de la clase de E/LE. 
c. Despertar en el alumnado el interés sobre el control de su afectividad y su importancia para el buen desarrollo en el aprendizaje de E/LE.

d. Clarificar directrices de actuación para el profesorado de este grupo-curso con el fin de potenciar el componente afectivo-existencial de la lengua extranjera.

\subsection{Contexto}

Se eligió para la presente investigación un Centro de Lenguas Público (CIL) en el Distrito Federal de Brasil. Las clases tienen una duración de una hora y 20 minutos, con una frecuencia equivalente a dos veces a la semana. La escuela está constituida por 12 aulas equipadas con televisor, vídeo y reproductor de sonido.

Según la "Orientación Pedagógica" (2002), documento que normaliza todos los Centros de Lenguas Públicos de Brasilia (CILs), se debe adoptar una metodología dinámica y con un "enfoque comunicativo" para que el alumno sea capaz de expresarse, de entender, de interpretar y de comunicarse con su interlocutor. En este mismo documento se considera que las estrategias construyen y rescatan la confianza de los alumnos, eliminando barreras que puedan impedir el aprendizaje. Además, la metodología puede ser adaptada a las especificaciones de cada grupo.

El sistema de enseñanza adoptado es semestral y por niveles y éstos en cuatro ciclos: Juvenil, Básico, Intermedio y Avanzado. Cada ciclo está compuesto de dos, cinco, cuatro y tres semestres respectivamente.

\subsection{Informantes}

La muestra elegida para el estudio empírico es de 17 estudiantes de E/LE del nivel Intermedio (nivel B1 del MCERL). Todos son brasileños y llevan un promedio de 3 años estudiando el castellano. Se solicitó por escrito a los estudiantes que participaran en la presente investigación y todos firmaron aceptándolo. A continuación describiremos a los informantes de la investigación cuyos datos personales fueron protegidos bajo pseudónimos elegidos por ellos mismos.

Tabla I. Alumnado informante para el estudio.

\begin{tabular}{|c|c|c|c|c|}
\hline PSEUDÓNIMO & EDAD & GÉNERO & PROFESIÓN & $\begin{array}{l}\text { TIEMPO } \\
\text { ESTUDIANDO } \\
\text { ESPAÑOL }\end{array}$ \\
\hline ANA & 27 & Mujer & Abogada & 3 años \\
\hline ANE & 23 & Mujer & Estudiante & 2 años \\
\hline EDU & 21 & Hombre & Estudiante & 3 años \\
\hline FURICO & 19 & Hombre & $\begin{array}{l}\text { Estudiante y trabajador en } \\
\text { prácticas }\end{array}$ & 3 años y medio \\
\hline
\end{tabular}


Afectividad y competencia existencial en estudiantes de español como... / P. R. Marins De ANdrade, J. R. GuijarRo OjEDA

Continuación Tabla I.

\begin{tabular}{|c|c|c|c|c|}
\hline GURIA & 17 & Mujer & Estudiante & 3 años \\
\hline JESÚS & 21 & Hombre & $\begin{array}{l}\text { Estudiante y trabajador en } \\
\text { prácticas }\end{array}$ & \\
\hline K TATAL & 19 & Hombre & Parado & 3 años y medio \\
\hline LAURA FERRARI & 18 & Mujer & Estudiante & 3 años y medio \\
\hline LOLA & 25 & Mujer & Psicóloga & 3 años \\
\hline MACHADO & 28 & Mujer & Administradora & 3 años \\
\hline MARI & 16 & Mujer & Estudiante & 3 años \\
\hline MARGI SIMPSON & 17 & Mujer & Estudiante & 3 años \\
\hline PILAR & 21 & Mujer & Estudiante & 3 años \\
\hline JUAN & 47 & Hombre & Profesor de la Red Pública & 3 años \\
\hline SIMBA & $\begin{array}{l}\text { No responde } \\
\text { (mediana edad) }\end{array}$ & Mujer & Técnica de laboratorio & 3 años \\
\hline TATA & 22 & Mujer & Estudiante & 3 años \\
\hline VAMPIRA & 19 & Mujer & Estudiante & 3 años \\
\hline
\end{tabular}

\subsection{Instrumento}

\subsubsection{Cuestionario}

El cuestionario se aplicó tanto al alumnado como al profesorado de la institución educativa en cuestión, aunque aquí solamente presentamos los resultados de las respuestas del alumnado para cumplir con los objetivos planteados en este estudio.

La elaboración del cuestionario está basada en las teorías revisadas sobre cómo inciden las variables afectivas en la clase de E/LE y los beneficios o desventajas que éstas pudieran tener sobre el aprendizaje de la lengua. Con el fin de fijar su validez, el instrumento fue sometido al juicio de tres expertos del área en el plano de la didáctica de la lengua extranjera, de los métodos de investigación en educación y de la didáctica general focalizada en la investigación cualitativa. El juicio de los expertos concluye que se trata de un cuestionario pertinente para dilucidar información valiosa del alumnado en torno a la temática planteada, tratándose del estudio a micronivel de un contexto muy específico en Brasilia.

Con el propósito de alcanzar los objetivos previstos se concretaron finalmente las siguientes preguntas para ser contestadas por el alumnado:

1. ¿Crees que la clase de español es un ambiente favorable para aprender esa lengua y además, un lugar donde puedes compartir tus esperanzas, tus sueños y tus ambiciones, tus experiencias, recuerdos y deseos, tus intereses, sentimientos, cualidades, valores e ideas?

a. De acuerdo; b. Parcialmente de acuerdo; c. En desacuerdo. 
2. ¿Qué actividades pueden mejorar tu autoestima? ¿Por qué?

a. Actividades con música; b. Debates dirigidos; c. Dramatizaciones; d. Tutoría personalizada; e. Tratamiento personalizado al final de clase.

3. ¿Cuál es tu mayor frustración como estudiante de español?

a. No tener tiempo para estudiar en casa; b. No conseguir hablar lo que quiero cuando quiero por factores emotivos; c. No tengo frustraciones; d. No obtener las notas que me gustaría; e. No consigo aprender como me gustaría; f. Aunque tuviera tiempo no estaría más de lo necesario.

4. ¿Dónde crees que debe estar el foco del proceso de enseñanza/aprendizaje?

a. En el profesor, su enfoque y método utilizados en clase; b. En los alumnos y en cómo aprender; c. En las actividades y evaluaciones; d. En las variables afectivas.

5. ¿En qué actividades te sientes más emocionalmente involucrado/involucrada? ¿Por qué?

a. Tareas de expresión oral; b. Tareas de comprensión lectora; c. Tareas de expresión escrita; d. Tareas de expresión oral.

6. ¿Crees que las actividades desafiadoras motivan o desaniman? ¿Por qué?

a. Motivan; b. Desestimulan; c. Depende de otros factores; d. Igualmente importantes.

7. ¿Qué es lo que más te desmotiva al aprender español?

8. ¿Te producen ansiedad las actividades desafiadoras?

9. ¿Qué tipo de tareas son más motivadoras para ti y cuáles te desmotivan más?

10. ¿En qué momento te sentiste mal, incómodo o amenazado durante este semestre?

\section{ANÁLISIS Y DISCUSIÓN DE LOS RESULTADOS}

En el estudio empírico se preguntó al alumnado informante sobre el conocimiento y control de los factores afectivos y su repercusión en el proceso de aprendizaje de E/LE y se llegaron a los resultados que se presentan a continuación y que han sido analizados a la luz de las teorías estudiadas. 


\subsection{Resultados de las preguntas del cuestionario}

1) ¿Crees que la clase de español es un ambiente favorable para aprender esa lengua y además, un lugar donde puedes compartir tus esperanzas, tus sueños y tus ambiciones, tus experiencias, recuerdos y deseos, tus intereses, sentimientos, cualidades, valores $e$ ideas?

Gráfico 1. La clase de E/LE como contexto afectivo favorable.

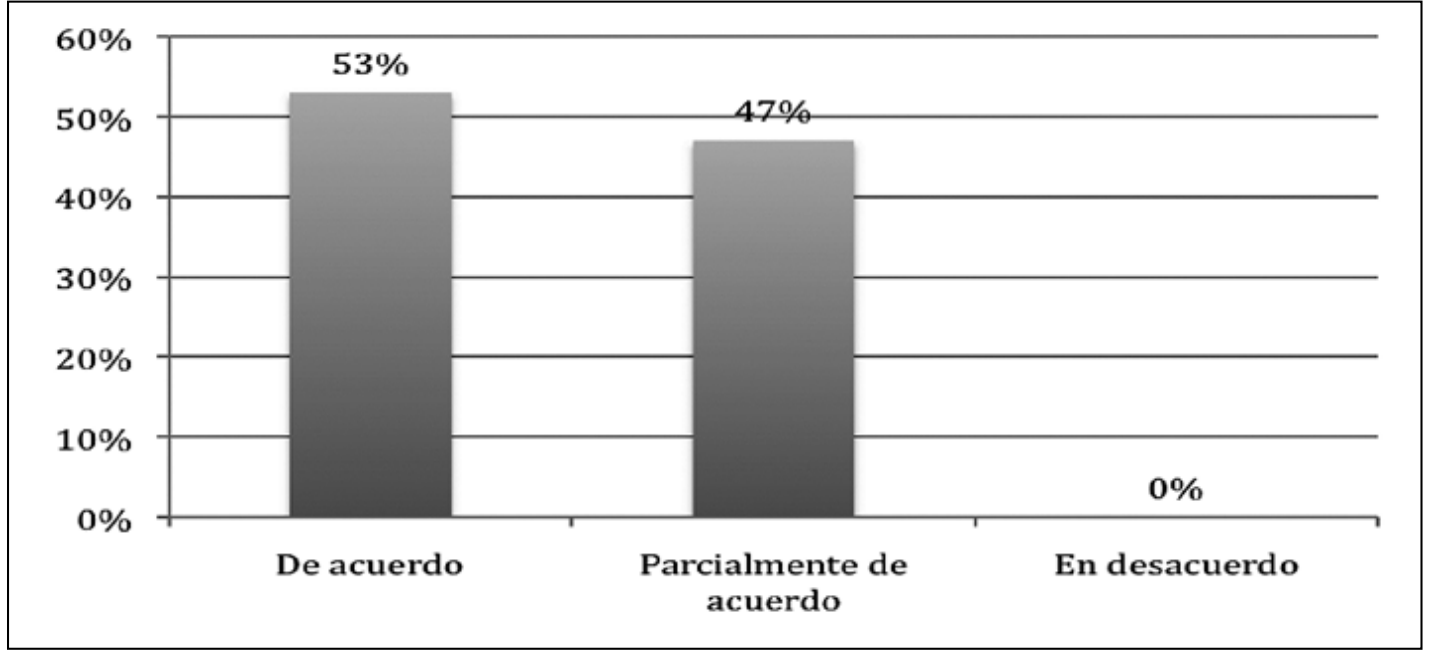

Estas respuestas demuestran que la mayoría de los estudiantes (53\%) considera que las variables emocionales (sentimientos, esperanzas, etc.) juegan un papel predominante en la clase de idiomas. Aunque una minoría considerable $47 \%$ está en parcial desacuerdo; por otro lado, nadie está en completo desacuerdo. Es decir, para un poco menos de la mitad de los estudiantes entrevistados el componente afectivo tiene una cierta relevancia en el aula de E/LE, aunque parece no ser el eje central de la docencia, visión ésta que debe ser trabajada con vistas a su cambio. Inversamente, un poco más de la mitad reconoce la importancia de los factores de orden afectivo y que éstos deben ser considerados en la clase de E/LE además de los factores de orden cognitivo. De los resultados obtenidos se evidencia la necesidad de un tratamiento didáctico más profundo en el aula, al igual que la labor concientizadora de la importancia que debe ejercer el profesorado por inculcar al alumnado la relevancia de potenciar en el aula el binomio afectividad-cognición en aras de un mejor andamiaje del aprendizaje para que sea más efectivo y acelerado. 


\section{2) ¿Qué actividades pueden mejorar tu autoestima? ¿Por qué?}

Gráfico 2. Actividades que mejoran la autoestima.

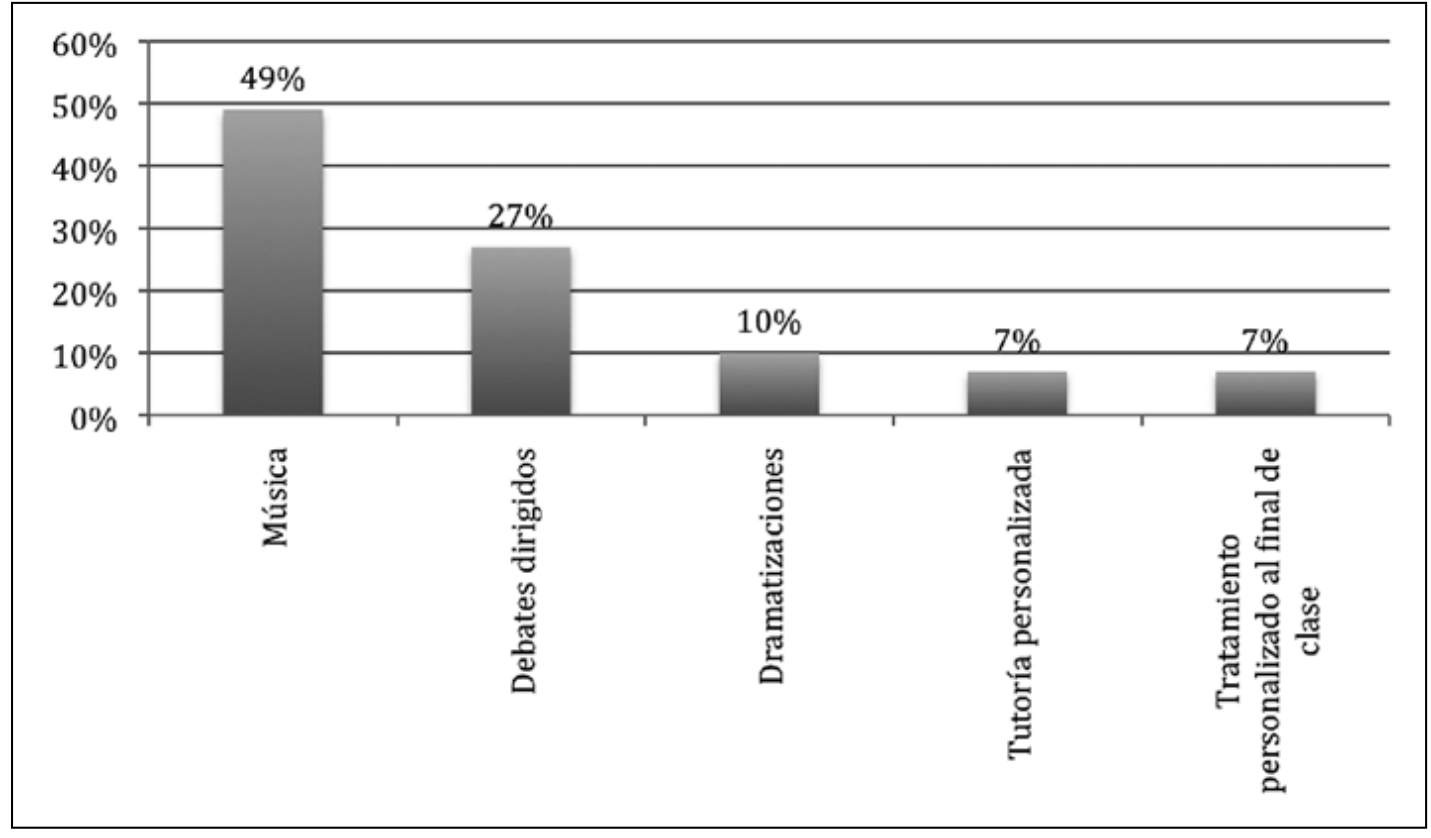

La mayoría ha marcado más de una alternativa. No obstante, se nota que han predominado las actividades con música entre las preferidas para mejorar la autoestima (49\%) seguidas de los debates dirigidos (27\%). Cabe destacar que tutorías y tratamiento personalizado al final de la clase gozan de poco prestigio entre el alumnado informante.

Para contestar a los porqué agrupamos las respuestas por similitudes: Ana, Mari y Vampira consideran que la música las relaja, las tranquiliza y las alivia. Guria y Margi Simpson dicen que les gusta cantar incluso Margi confiesa que a ella le gustaría ser una cantante. Pilar y Vampira dicen que las actividades musicales son alegres y divertidas, por lo tanto, relajantes. Ane cree que las canciones le facilitan el aprendizaje de la lengua española. Juan cree que las canciones son un buen estímulo pues aportan un estilo metodológico diferente a las clases. Laura Ferrari y Simba creen que los debates son más productivos y, además, Simba dice que le ayudan a no olvidar lo aprendido en las clases. Machado y Tata simplemente dicen que les gusta la música pues les interesa.

Los resultados nos llevan a plantear nuevas perspectivas metodológicas para que el alumnado pueda sentir más afinidad afectiva con las tareas propias de la 
enseñanza-aprendizaje de E/LE. Tanto actividades con música/canciones como los debates dirigidos han de ser potenciados, avalados en el ámbito internacional por su potencial lingüístico y comunicativo, añadiendo aquí la relevancia de su componente afectivo-existencial.

\section{3) ¿Cuál es tu mayor frustración como estudiante de español?}

Gráfico 3. Frustraciones como estudiante de E/LE.

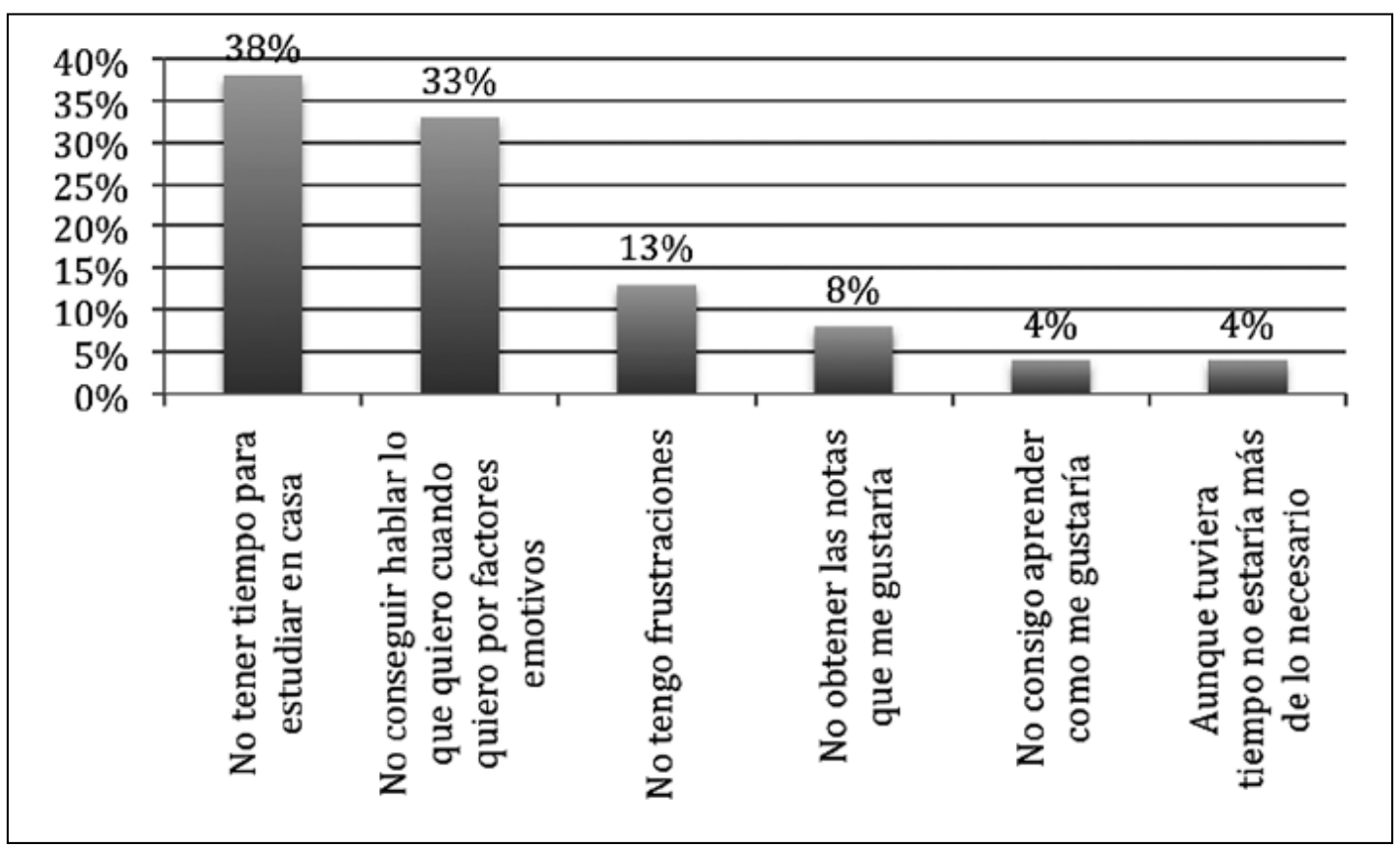

Para los estudiantes "no tener tiempo suficiente para estudiar en casa" es su mayor frustración, lo que desencadena, en consecuencia, la segunda opción que es "no conseguir hablar lo que quiere cuando quiere por factores emotivos". Una vez más reconocen que la afectividad interfiere de manera negativa en su desarrollo oral por no conseguir controlarse y por tener baja Inteligencia Emocional. Esto nos hace deducir que los factores emotivos interfieren preponderantemente en su competencia comunicativa, el hecho de hablar está condicionado por la emoción, además, los aprendices se mostraron conscientes de ello.

Por las características propias del centro estudiado es evidente que muchos alumnos son trabajadores, por lo que es una variable fundamental para entender que la falta de tiempo es una característica que frustra, lo que genera un alto índice de ansiedad y dificulta el aprendizaje de la lengua. 


\section{4) ¿Dónde crees que debe estar el foco del proceso de enseñanzalaprendizaje?}

Gráfico 4. El foco del proceso de enseñanza/aprendizaje.

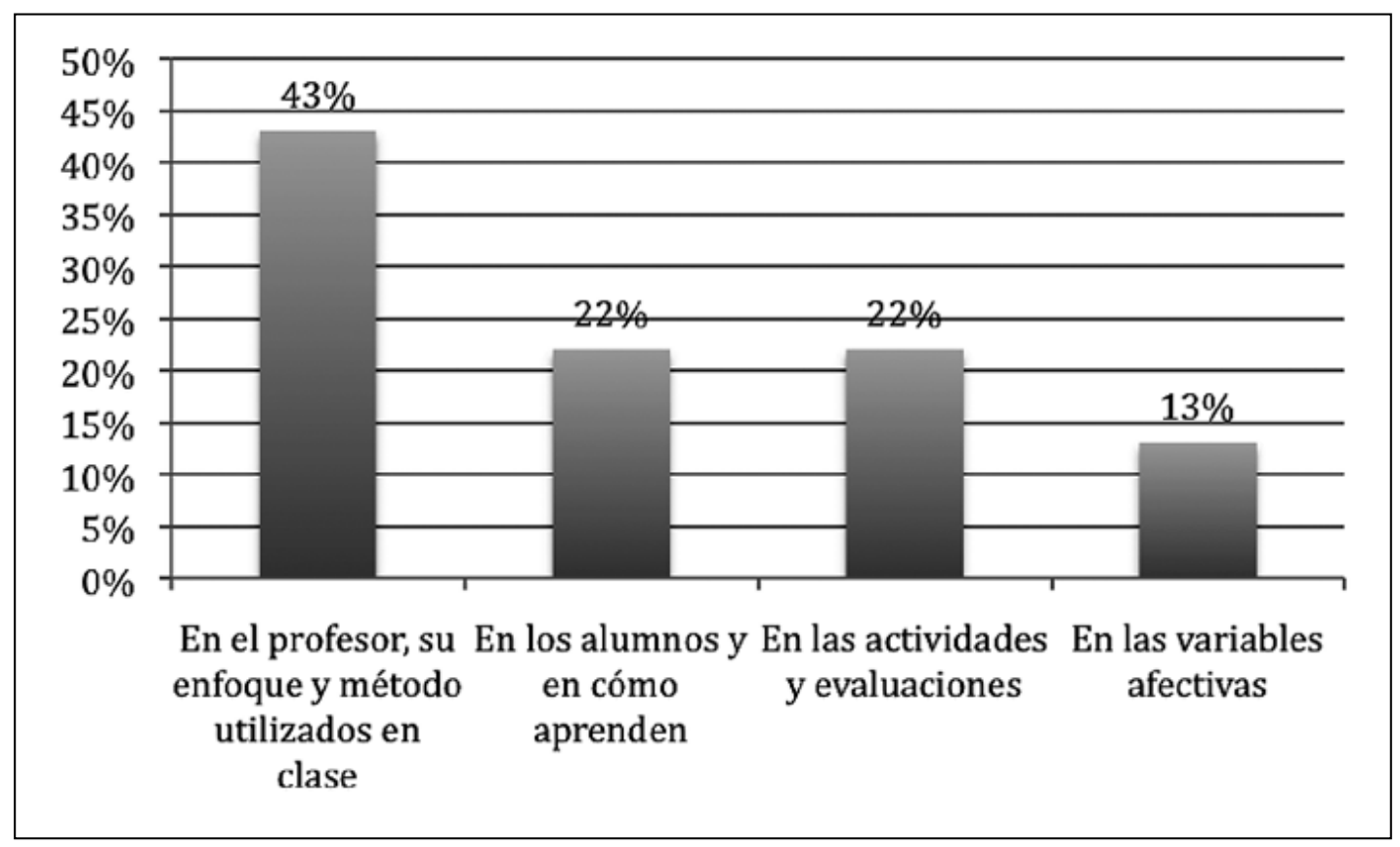

La mayor parte de los alumnos ha señalado al profesor/profesora, su enfoque y métodos utilizados como principal foco del proceso de enseñanza/aprendizaje, es decir, para ellos, el proceso debe estar centrado en el docente como principal protagonista. Por todo ello, estas respuestas demuestran que los estudiantes tienen una visión más bien tradicional de la enseñanza/aprendizaje a pesar de ser importante la correcta elección del método/enfoque. Un 22\% ha marcado "en las actividades y evaluaciones", otro $22 \%$ "en los alumnos y en cómo aprenden". Sólo un $13 \%$, la minoría, ha señalado que las variables emocionales juegan también un papel fundamental. Todavía no tienen una visión individual del proceso como el tratamiento individualizado y personalizado teniendo en cuenta las diferencias individuales y más bien una visión colectiva (el grupo). Al igual que Reid (2000) abogamos por la necesidad de que el alumnado pueda tener conciencia de la afectividad en el aula para que pueda mejorar su propio desempeño, forjando así, los cimientos sobre los cuales construirá su aprendizaje teniendo como consecuencia la mejora de su propio desempeño como aprendiz de lenguas. 
5) ¿En qué actividades te sientes más involucrado emocionalmente? ¿Por qué?

Gráfico 5. Implicación emocional en actividades.

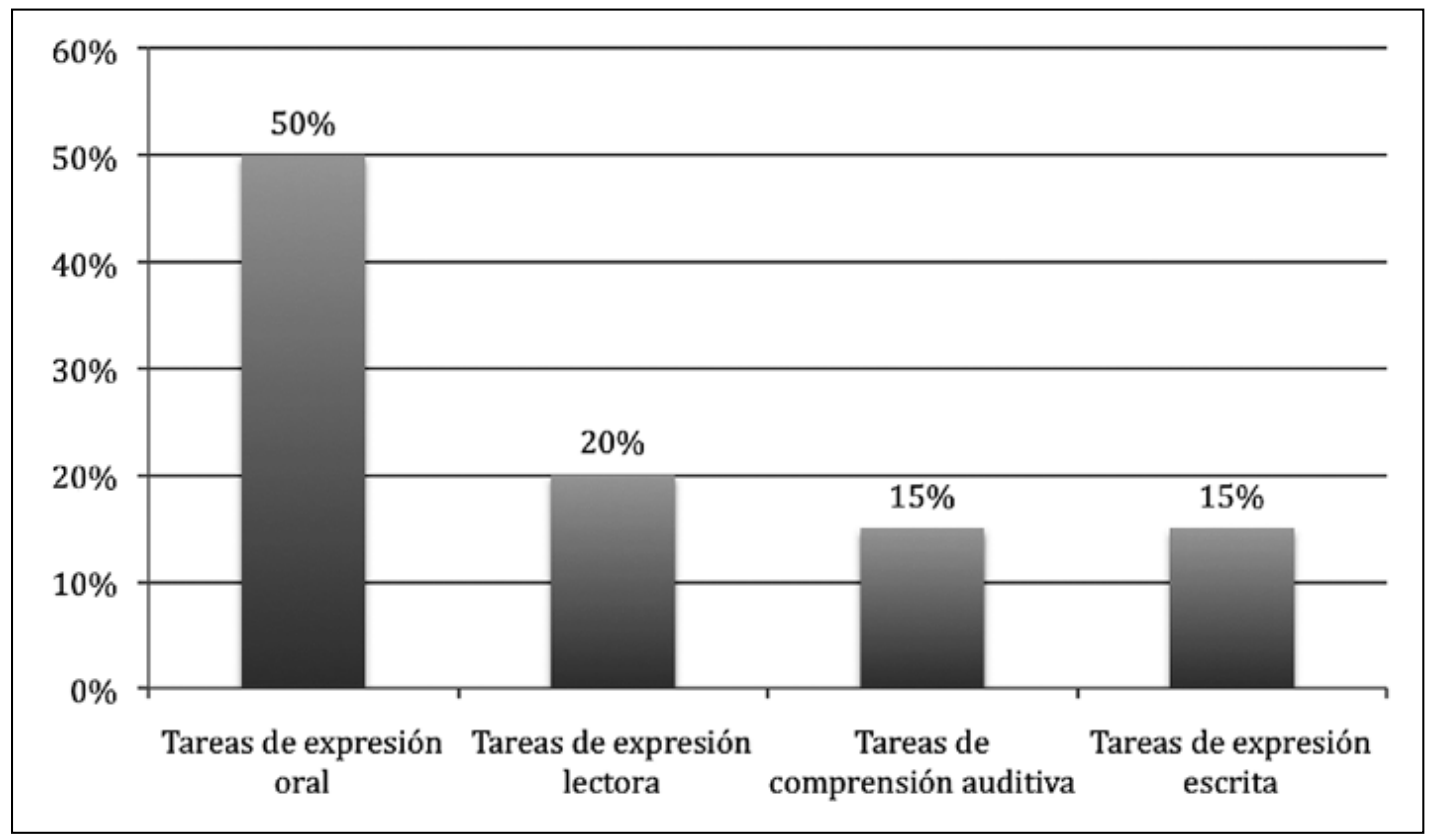

$\mathrm{Al}$ apuntar las tareas de expresión oral como en las que la afectividad juega un papel decisivo, se deduce que ésta es la razón por la cual a muchos profesores y profesoras no les gusta trabajar bajo un enfoque "conversacional", por así decirlo, a fin de evitar trastornos o ansiedades en la clase. No obstante, creemos que al provocar ansiedad en los estudiantes a la hora de hablar el docente debe saber cómo trabajarla por medio de las estrategias afectivas haciendo uso de las propuestas de Oxford (1990) como las técnicas de relajación, el uso de la música, el humor, escuchar el cuerpo o el hecho de "componer un diario". Este último puede funcionar muy bien como una "catarsis" o como una válvula de escape, para descargar todas sus frustraciones y todo aquello que no se pudo expresar "oralmente" en clase por cuestiones emotivas.

Al contestar por qué se considera la expresión oral como destreza con más influencia emocional éstas fueron las respuestas: Lola cree que la expresión oral es una tarea más completa sobre todo en debates: "Porque en estas tenemos que oír y comprender y también contestar", en realidad se refiere a la "interacción oral" - lo que es más difícil, pues requiere el uso de otras destrezas (oír, comprender, interpretar - intencionalidad) y contestar (expresión oral). Guria cree que en los exámenes orales es una responsabilidad dar su opinión sin contradecirse. Machado dice que aunque cometa errores intenta hablar más en español. Pilar cree que las tareas de expresión y comprensión oral son las que exigen más conocimiento y esfuerzo 
por parte del alumno, sobre todo cuando uno está inseguro o no familiarizado con la lengua. Mari destaca la dificultad de la tarea oral para ella pues es tímida.

Otras respuestas con relación a otras destrezas fueron: a Ana le encanta leer cuentos, libros e historias. Furico cree que las tareas de expresión escrita le estimulan a escribir más y a cometer menos errores además de poder expresar su opinión. Vampira dice que las tareas de comprensión auditiva son mejores pues le ayudan a hablar y a comprender lo que hablan. Juan destaca la importancia del léxico y de memorizar "más palabras", destacando que ésa es la forma en que todas las tareas deberían funcionar para una adquisición más correcta. Simba cree que las tareas de comprensión auditiva le cohíben emocionalmente ya que se habla rápido y, para ella, esto es un factor afectivo desfavorecedor o frustrante.

\section{6) ¿Crees que las actividades desafiadoras motivan o desaniman? ¿Por qué?}

Gráfico 6. Poder motivador de actividades desafiadoras.

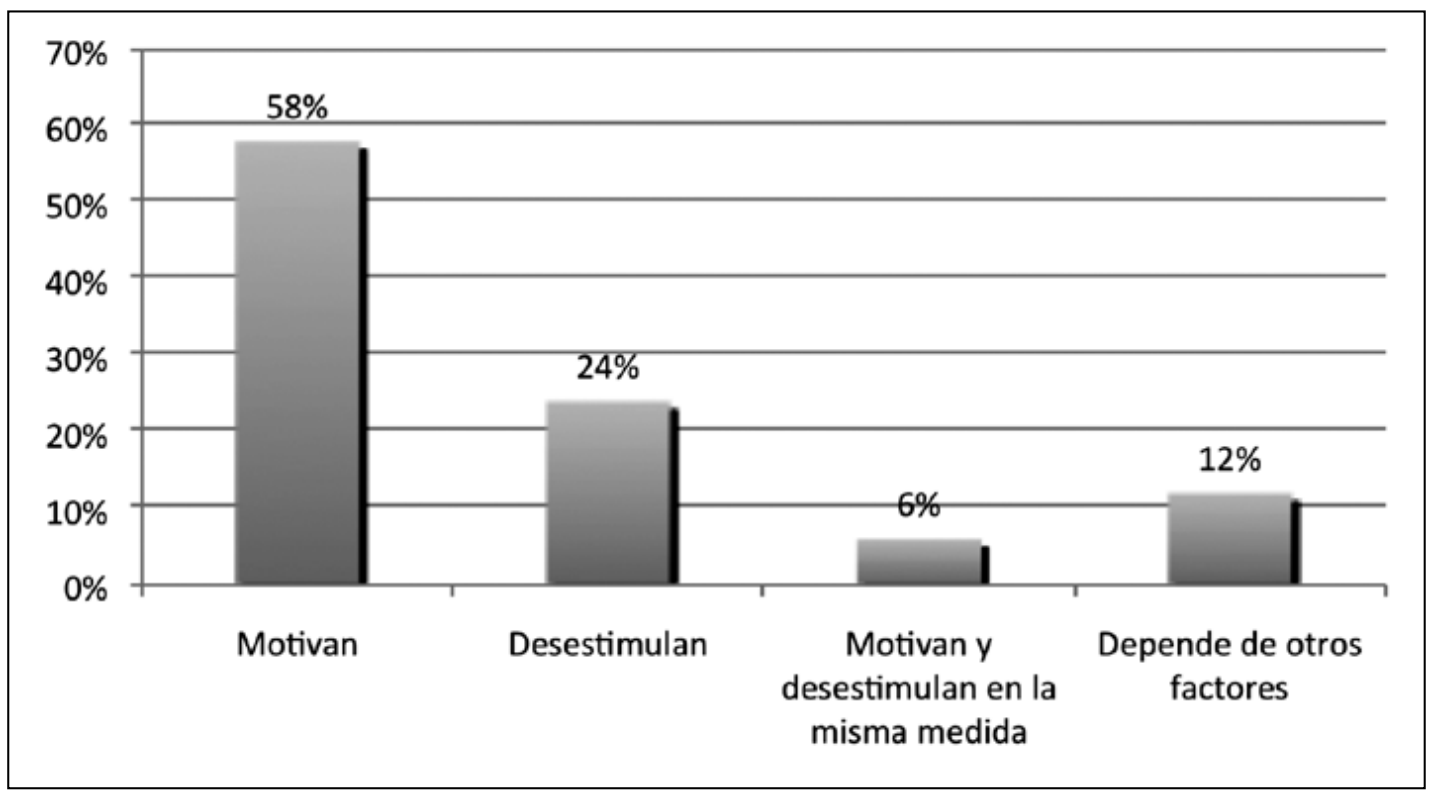

Al hablar de la motivación en actividades desafiadoras, la mayoría ha dicho que motivan. Los que así lo creen han dicho que estas actividades les hacen prepararse mejor, estudiar e investigar más, descubrir sus límites para superarlos, y esto constituye una forma de crecimiento y de aprendizaje. Estas fueron algunas de las respuestas sobre el porqué:

Para Ana las actividades desafiadoras motivan tan sólo si es posible realizarlas, es decir, el nivel de desafío debe ajustarse al nivel de los/las estudiantes y sus capacidades cognitivas, ya que si no es así puede repercutir negativamente resultando en la falta de motivación y consecuente evasión como lo corrobora Simba al afirmar 
que "cuando no se entiende [la actividad] no nos motiva a seguir adelante". Por lo tanto, todo depende del estado de ánimo y disposición de los aprendices. Para Pilar, las actividades desafiadoras generan un aumento del nivel de autonomía pues la llevan a "buscar más tareas extra-clase para mejorar el desempeño". Lola cree que los objetivos de la actividad deben estar claros para que sea motivadora. Jesús dice que las actividades desafiadoras "desestimulan” pues provocan ansiedad. Es decir, él no cree en los beneficios que puede traernos un desafío (producto) sino en su repercusión durante el proceso (ansiedad). Es decir, la respuesta está en la forma en que el docente debe conducir la actividad de manera más amena aunque constituya un desafío, buscar estrategias para superarlos. K Tatal y Mari afirman tajantemente que les gusta enfrentarse a desafíos.

\section{7) ¿Qué es lo que más te desmotiva al aprender español?}

Los estudiantes señalan tres factores principales como los causantes de mayor desmotivación: el horario de las clases (nocturno), la gramática (considerada "difícil") y la falta de tiempo para estudiar y hacer las tareas. No obstante, Machado y Furico dicen que es aburrido estudiar la gramática de cualquier lengua.

Lola y Guria casi coinciden en sus respuestas. Lola dice que se desmotiva pues no está en un contexto de inmersión y Guria también considera que la propuesta del curso no es muy original y que una clase por semana es muy poco.

Además, hubo respuestas muy diferentes por parte de otros alumnos. Por ejemplo, Simba ha dicho que se siente desmotivada al aprender español pues lo considera un desafío, así para ella los desafíos la desmotivan. Vampira confiesa que lo que más la desmotiva son las profesoras.

Las respuestas confirman como elementos desmotivadores los tradicionalmente indicados en estudios internacionales: la gramática, el profesorado y las constricciones del tiempo para el estudio, incluido el horario de las clases.

\section{8) ¿Te producen ansiedad las actividades desafiadoras? ¿Por qué?}

La mayoría ha señalado que "sí" producen mucha ansiedad (11 alumnos). No obstante, 5 alumnos confesaron que "no", que no le producían ansiedad. Juan y Mari han dicho que ya son personas muy ansiosas por naturaleza, así que una situación desafiante les aumenta la ansiedad. Aquí la mayor parte de los estudiantes comparten la misma opinión sobre lo que más puede provocar ansiedad son los proyectos. Los aprendices destacan que, a pesar de producir ansiedad, puede ser motivador al mismo tiempo. Laura Ferrari dice que se pone nerviosa pues no le dedica mucho tiempo, así que le parece que no habla como debiera considerando el tiempo de estudio que lleva, lo que evidencia la necesidad por su parte de trabajar la autoestima. 
Entre los que respondieron "no" tenemos: Vampira y Machado dijeron que las actividades desafiantes los motivan. Machado dice que siempre es bueno tener un desafío para comprobar sus límites. Comparte la misma opinión Guria que dice que le gustan las actividades con incentivo. Lola dice que las actividades desafiantes tienen que tener un objetivo claro. Sugiere que hay que unir la práctica con la teoría, por ejemplo, si van a hablar, tienen que usar lo que han aprendido en la gramática. Así se concluye que a la mayoría de los estudiantes les producen ansiedad las actividades desafiantes. Entre todos, tanto los que dijeron que sí como los que no, algunos destacan el aspecto positivo de la ansiedad, es decir, la ansiedad buena, pues les hace investigar más, aprender cosas buenas, examinar sus límites, es decir, que los motiva. Todo esto confirma la teoría de Omaggio Hadley (2000). Oxford (2000) relaciona la ansiedad con otros aspectos como la autoestima. Es lo que ocurre con Laura Ferrari, que, por no estar segura de sí misma en el dominio del idioma, se siente ansiosa.

\section{9) ¿Qué tipos de tareas son más motivadoras para ti y cuáles te desmotivan más?}

Se pudo observar que en cuanto a las tareas desmotivadoras casi todos apuntaron las actividades que envuelven la gramática utilizada o cualquier actividad gramatical. De entre las motivadoras, gran parte de los entrevistados apuntaron las actividades con música y las actividades orales. Sin embargo, se observó que lo que puede ser motivador para uno es desmotivador para otro alumno, como es el caso de Lola pues, para ella, la práctica de la gramática es muy motivadora, al contrario de la mayoría. Para Lola las redacciones son desmotivantes y a Mari, la motivan. Así como Juan y Jesús: para el primero leer libros es muy motivador y para el segundo los libros lo desmotivan. Para Edu y Ane las actividades orales y debates son motivadores y para Laura Ferrari los debates son desmotivantes. Es decir, hay una unanimidad dentro de una diversidad de opiniones opuestas.

10) ¿En qué momento te sentiste mal, incómodo, o amenazado durante este semestre?

El 46\% apuntaron prioritariamente factores como el cansancio, el trabajo y las evaluaciones como los más amenazadores o causantes de malestar durante las clases. Los otros 16\% destacaron factores diversos, entre ellos las dinámicas que no han funcionado pues en lugar de "quitar el dolor" del aprendizaje les provocó nerviosismo. Un porcentaje significativo, el 38\%, declara que no tuvieron problemas emocionales durante las clases.

\subsection{Discusión de resultados}

A la luz de los resultados, en primer lugar es imperativo destacar que todo lo 
que conforma el término "competencia existencial" guarda un fuerte vínculo o relación con las variables afectivas investigadas. Además, el término "competencia comunicativa" tiene una relación unívoca con la "competencia de aprendizaje" y con la "competencia existencial" pues el hecho de hablar (tener algo que decir) está condicionado por la emoción. Por otro lado, factores como la personalidad y el tipo de actividades inciden en la capacidad para aprender, capacidad ésta que está estrechamente vinculada con la autonomía y las estrategias.

La relación entre emociones y cognición es nítida pues los sentimientos pueden incluso despertar la autonomía en el aprendizaje. Además, la eficacia de habilidades cognitivas está condicionada al estado emocional por lo que se aboga por el aprendizaje del autocontrol y del dominio de las propias emociones (inteligencia emocional). Así es que el autoconocimiento conduce a un autocontrol (control de las emociones) resultando en el aumento de la autoestima y de la automotivación.

Una de las premisas que supone la competencia existencial es la capacidad para desarrollar la motivación. Por esta razón, el papel del profesor como fuente de motivación es primordial, debiendo, para tal fin, estar motivado él mismo. Por otro lado, el profesorado de lenguas extranjeras debe ser sensible para no frustrar la autoestima del alumno controlando demasiado su actuación y podando su libertad de expresión auténtica. El desarrollo de una personalidad empática puede favorecer el de la competencia comunicativa intercultural. Además, desarrollando el Coeficiente Emocional del alumno, el docente estaría capacitándolo para ser más empático.

\section{CONCLUSIONES}

Tras el planteamiento teórico y empírico abordado en este trabajo hemos podido estudiar, analizar y evaluar los resultados de algunos aspectos del proceso de enseñanza-aprendizaje de E/LE con relación a los factores afectivo-emocionales del alumnado de un Centro Superior de Lenguas del Distrito Federal de Brasil. Las conclusiones que se han obtenido satisfacen los objetivos planteados para esta investigación.

Nuestro primer objetivo consistía en conocer las opiniones del alumnado-meta sobre aspectos afectivo-existenciales que intervenían en su aprendizaje de E/LE. $\mathrm{Al}$ respecto podemos señalar como principal conclusión la poca importancia con que es tratado el tema de la afectividad en los procesos de enseñanza-aprendizaje de lenguas extranjeras hoy en día en el contexto estudiado, considerando las apreciaciones del alumnado.

En segundo lugar, deseábamos saber el grado en que variables afectivo-emocionales tales como el control de la ansiedad, el desarrollo de la autonomía y de 
la autoestima o la motivación pueden facilitar o entorpecer la solución de los desafíos de la clase de E/LE. En relación a este objetivo, podemos concluir que la mayoría de los entrevistados considera que la música les mejora la autoestima pues les alivia, relaja, divierte, les facilita el aprendizaje, además de ser estimulante y hacerles cantar. Al mismo tiempo, un alto índice de alumnos destacaron como actividades muy motivadoras las películas y las actividades orales. Como actividades desmotivadoras podríamos apuntar las tareas gramaticales. La afectividad interfiere de manera negativa en su desarrollo oral por no tener autocontrol o baja Inteligencia Emocional. La expresión oral es la destreza con mayor influencia emocional, ya que provoca más ansiedad; lo que demanda la implementación de estrategias afectivas por parte del profesorado.

Despertar en el alumnado el interés sobre el control de su afectividad y su importancia para el buen desarrollo en el aprendizaje de E/LE, se erigía como el tercero de los objetivos de este trabajo. En este orden de cosas, la mayoría de los informantes aún considera al profesor como el protagonista del proceso de enseñanza-aprendizaje. La mayoría cree que los desafíos les provoca ansiedad, no obstante pueden ejercer al mismo tiempo un aliento motivador, pues es enfrentándolos cuando se preparan mejor, estudian e investigan, propiciando su crecimiento no sólo cognitivo sino también afectivo. No obstante, hemos podido constatar que el propio proceso de investigación ha servido para replantear y traer a la arena del debate la importancia capital que tiene el control de la propia afectividad para tener éxito en el aprendizaje de una lengua extranjera.

Por último, y con fines marcadamente didácticos, nos proponíamos apuntar directrices de actuación para el profesorado de este grupo-curso con el fin de potenciar el componente afectivo-existencial de la lengua extranjera. Las apreciaciones del alumnado son muy clarificadoras al respecto y las que interfieren negativamente en los factores afectivos del alumnado estudiado son: dinámicas didácticas mal empleadas; evaluaciones no ajustadas a los objetivos planteados que provocan malestar y sentimiento de incomodidad; actividades gramaticales como fin en sí mismas, lo que dificulta el componente comunicativo y lúdico de la lengua; falta de tiempo para la realización de tareas junto al horario complicado de las clases. Siendo así, estas cuestiones han de ser paliadas por el profesorado responsable de los cursos.

Concluyendo, a través de este estudio deducimos que se hacen necesarios más estudios comparativos profundos sobre la relación que hay entre "metodologías" y "variables afectivas" para, según los resultados que se obtengan, comprobar la eficacia/ineficacia del método teniendo en cuenta los factores internos. Por otro lado, no se debe perder el foco del proceso: el aprendizaje y que éste se realice de una manera más agradable y eficaz a la vez, evitando así los sufrimientos innecesarios y sacando provecho de los conflictos. De este modo, la incorporación de estrategias afectivas en el plan de clase de cualquier profesor convertiría su forma de enseñar/ 
aprender en un proceso dialógico más educativo y completo, teniendo en cuenta los intereses y afectividad del alumnado.

\section{REFERENCIAS}

Acosta Padrón, R. y Fernández de Lara Arias, C. 1996. "Concepción Integradora para la enseñanza lenguas”, en Cuadernos Cervantes, 10, pp. 44-45.

Alonso Tapia, J. y Cartula Fita, E. 2003. A motivação em sala de aula: o que é como se faz. São Paulo: Loyola.

Andrés, V. 2000. "La autoestima en el aula o la metamorfosis de las mariposas", en J. Arnold. La dimensión afectiva en el aprendizaje de idiomas. Madrid: CUP.

Arnold, J. 2000. La dimensión afectiva en el aprendizaje de idiomas. Madrid: CUP.

Arnold, J. y Brown, D. 2000. "Mapa del Terreno”, en J. Arnold. La dimensión afectiva en el aprendizaje de idiomas. Madrid: CUP.

Consejo de Europa. 2002. Marco Común Europeo de referencia para las lenguas: aprendizaje, enseñanza, evaluación. Estrasburgo: Language Policy Division. (traducción al español: Instituto Cervantes, 2002).

Gardner, H. 2007. "Motivation and second language acquisition”. En Porta Linguarum, 8, pp.5-25.

Gardner, R.C. y MacIntyre, P.D. 1993. "On the measurement of affective variables in second language learning". En Language Learning, 43, pp. 157-194.

Giovannini, A. 2000. Profesor en acción, 1 - El proceso de aprendizaje. Madrid: Edelsa.

Goleman, D. 1997. Inteligencia Emocional. Barcelona: Kairós.

Guijarro Ojeda, J. R. 2004. Attitudes and Motivation in Second and Foreign Language Learning. Granada: Reprografía Digital.

Guijarro Ojeda, J. R. 2007. "Análisis de los elementos axiológicos en la normativa española para el Inglés como Lengua extranjera”. En Cauce. Revista Internacional de Filología y sus Didácticas, 30, pp. 147-164.

Horwitz, E. K. 1986. "Preliminary evidence for the reliability and validity of a foreign language Anxiety Scale”. En TESOL Quarterly, 20, pp. 559-562.

Jiménez Luna, E. 2005. Los factores afectivos en las programaciones de cursos. La motivación. Memoria de Máster no publicada. Madrid: Universidad Antonio de Nebrija.

Krashen, S. 1982. Principles and Practice in Second Language Acquisition. New York: Pergamon Press.

Omaggio Hadley, A. 2000. Teaching Language in Context. New York: Heinle.

Oxford, R. 1990. Language Learning Strategies. What every teacher should Know. Boston: Heinle \& Heinle Publishers. 
Oxford, R. 2000. "La ansiedad y el alumno de idiomas: nuevas ideas", en J. Arnold. La dimensión afectiva en el aprendizaje de idiomas. Madrid: CUP.

Reid, J. 2000. "La afectividad en el aula: problemas, política y pragmática”, en J. Arnold. La dimensión afectiva en el aprendizaje de idiomas. Madrid: CUP.

Secretaria de Estado de Educação do Distrito Federal (2002). Orientação Pedagógica. Centros Interescolares de Línguas. Brasilia: FEDF/DP.

Trujillo Sáez, F. 2005. "En torno a la interculturalidad: reflexiones sobre cultura y comunicación en didáctica de la lengua". En Porta Linguarum, 4, pp. 23-40.

Waltz, G. y Bleuer, J. 1992. Students' Self- Esteem: A Vital Element of School Success, Ann Arbor, Michigan, Counseling and Personnel Services.

Williams, M. y Burden, R. L. 1999. Psicología para profesores de idiomas. Cambridge: CUP. 